\title{
DIFFERENTIAL ALGEBRAS ON $\kappa$-MINKOWSKI SPACE AND ACTION OF THE LORENTZ ALGEBRA
}

\author{
STJEPAN MELJANAC, SAŠA KREŠIĆ-JURIĆ, AND RINA ŠTRAJN
}

\begin{abstract}
We propose two families of differential algebras of classical dimension on $\kappa$-Minkowski space. The algebras are constructed using realizations of the generators as formal power series in a Weyl super-algebra. We also propose a novel realization of the Lorentz algebra $\mathfrak{s o}(1, n-1)$ in terms of Grassmann-type variables. Using this realization we construct an action of $\mathfrak{s o}(1, n-1)$ on the two families of algebras. Restriction of the action to $\kappa$-Minkowski space is covariant. In contrast to the standard approach the action is not Lorentz covariant except on constant one-forms, but it does not require an extra cotangent direction.
\end{abstract}

Keywords: $\kappa$-Minkowski space, Lorentz algebra,realizations,differential algebra, covariance

PACS numbers: 02.20.Sv, 02.20.Uw, 02.40.Gh

\section{INTRODUCTION}

Noncommutative (NC) geometry has been proposed for many years as a suitable model for unification of quantum field theory and gravity. Noncommutative spaces have been studied from many different points of view, including operator theory [1] and Hopf algebras [2]-24]. In particular, the notion of differential calculus on NC spaces has been studied in Refs. 4]-[14]. It is known that many classes of NC spaces do not admit differential calculi of classical dimensions which are fully covariant under the expected group of symmetries [25]. This quantum anomaly for differential structures is usually fixed by introducing extra cotangent directions.

In this paper we focus our attention to $\kappa$-Minkowski space. This is a Lie algebra type NC space which appears as a deformation of ordinary Minkowski space-time within the framework of doubly special relativity (DSR) [26]-33]. The symmetry algebra for DSR is obtained by deforming the ordinary Poincaré algebra into a Hopf algebra known as $\kappa$-Poincaré algebra [29]-32]. Different bases of $\kappa$-Poincaré algebra correspond to different versions of DSR theory [29]. The $\kappa$-deformed Poincaré algebra as deformed symmetry of 
the $\kappa$-Minkowski space-time inspired many authors to construct quantum field theories (see e.g. Refs. [33]-38]) and electrodynamics on $\kappa$-Minkowski space-time [39]-[41], or to modify particle statistics [42, [43].

Bicovariant differential calculus on $\kappa$-Minkowski space-time was considered by Sitarz in Ref. [7. He has shown that if the bicovariant calculus is required to be Lorentz covariant, then one obtains a contradiction with a Jacobi identity for the generators of the differential algebra. This contradiction is resolved by adding an extra cotangent direction (one-form) which has no classical analogue. Thus, the differential calculus in $3+1$ dimensions developed in Ref. [7] is five-dimensional. This work was generalized to $n$ dimensions by Gonera et al. in Ref. [8]. There have been several attempts to deal with this issue in $\kappa$-Euclidean and $\kappa$-Minkowski spaces [9]-[14]. In Ref. [12] Bu et al. constructed a differential algebra on $\kappa$-Minkowski space from Jordanian twist of the Weyl algebra and showed that the algebra is closed in four dimensions. In their approach they extended the $\kappa$-Poincaré algebra with a dilatation operator and used a coproduct of the Lorentz generators which is different from the one used in Ref. [7]. In Refs. [13] and [14] differential algebras of classical dimension on $\kappa$-Euclidean and $\kappa$-Minkowski spaces are constructed. In this approach one-forms are obtained from an action of a deformed exterior derivative on NC coordinates. Different deformations of exterior derivative and NC coordinates lead to different versions of differential calculus compatible with $\kappa$-deformation.

In the present work we propose new families of differential algebras (denoted $\mathfrak{D}_{1}$ and $\mathfrak{D}_{2}$ ) on $\kappa$-Minkowski space $\mathcal{M}_{\kappa}$ using realizations of the generators as formal power series in a Weyl super-algebra. We also present a novel realization of the Lorentz algebra $\mathfrak{s o}(1, n-1)$ in terms of Grassmann-type variables. This realization is used to define an action of $\mathfrak{s o}(1, n-1)$ on $\mathfrak{D}_{1}$ and $\mathfrak{D}_{2}$ which is consistent with commutation relations in $\mathfrak{D}_{1}$ and $\mathfrak{D}_{2}$ and Lorentz covariant on constant one-forms. Restriction of the action to $\mathcal{M}_{\kappa}$ is covariant, thus $\mathcal{M}_{\kappa}$ is an $\mathfrak{s o}(1, n-1)$-module algebra.

The paper is organized as follows. In Sec. 2 we discuss briefly a method for constructing differential algebras based on realizations of $\mathrm{NC}$ coordinates and exterior derivative as formal power series in a Weyl super-algebra. We construct two families of differential algebras $\mathfrak{D}_{1}$ and $\mathfrak{D}_{2}$ of classical dimension on $\kappa$-Minkowski space, and discuss their properties. We also show that by the same method the differential algebras in Refs. [7] and [15] can be constructed. In Sec. 3 we propose an action of $\mathfrak{s o}(1, n-1)$ on $\mathfrak{D}_{1}$ and 
$\mathfrak{D}_{2}$ using a realization of $\mathfrak{s o}(1, n-1)$ in terms of Grassmann-type variables. The action is not Lorentz covariant except on one-forms, but it does not require an extra cotangent direction as in Ref. [7]. When the action is restricted to $\kappa$-Minkowski space (which is a subalgebra od $\mathfrak{D}_{1}$ and $\mathfrak{D}_{2}$ ), then the Lorentz algebra acts covariantly on products of space-time coordinates which reproduces the well-know result of Majid and Ruegg [3]. In Sec. 4 a short conclusion and future outlook is given.

\section{Differential algebras ON $\kappa$-MinkOWSKi SPACE}

In this section we present the main points of the construction of differential algebras on $\kappa$-Minkowski space using realizations. First, we consider the differential algebra introduced by Sitarz [7] (see also Ref. [15]).

The $\kappa$-Minkowski space $\mathcal{M}_{\kappa}$ is an associative algebra generated by space-time coordinates $\hat{x}_{0}, \hat{x}_{1}, \ldots, \hat{x}_{n-1}$ satisfying the Lie algebra type commutation relations

$$
\left[\hat{x}_{i}, \hat{x}_{j}\right]=0, \quad\left[\hat{x}_{0}, \hat{x}_{j}\right]=i a_{0} \hat{x}_{j}, \quad a_{0} \in \mathbb{R} .
$$

By convention latin indices run from 1 to $n-1$, and greek indices run from 0 to $n-1$. A bicovariant differential algebra compatible with relations (1) was constructed by Sitarz in Ref. [7]. He has shown that if the differential algebra is also Lorentz covariant, then the smallest such algebra in $3+1$ dimensions is five-dimensional. One of its equivalent forms is given by

$$
\begin{array}{llrl}
{\left[\hat{\xi}_{0}, \hat{x}_{0}\right]=-i a_{0} \theta^{\prime}+i a_{0} \hat{\xi}_{0},} & {\left[\hat{\xi}_{0}, \hat{x}_{j}\right]=i a_{0} \hat{\xi}_{j},} \\
{\left[\hat{\xi}_{i}, \hat{x}_{0}\right]=0,} & {\left[\hat{\xi}_{i}, \hat{x}_{j}\right]=-i a_{0} \delta_{i j} \theta^{\prime},} \\
{\left[\theta^{\prime}, \hat{x}_{0}\right]=-i a_{0} \theta^{\prime},} & {\left[\theta^{\prime}, \hat{x}_{j}\right]=0,}
\end{array}
$$

where $\hat{\xi}_{\mu}$ is the one-form corresponding to $\hat{x}_{\mu}$, and $\theta^{\prime}$ is a one-form representing an extra cotangent direction that has no classical analogue. The one-forms $\hat{\xi}_{\mu}$ and $\theta^{\prime}$ anticommute. Let $\mathfrak{D}$ denote the algebra (2)-(4). The algebra $\mathfrak{D}$ was considered in Ref. [15] where it is shown that by gauging a coefficient of $\theta^{\prime}$ one can introduce gravity in the model. If we make a change of basis $\theta=\hat{\xi}_{0}-\theta^{\prime}$, we recover the original algebra introduced in Ref. [7]. As stated above, $\mathfrak{D}$ is constructed by postulating both bicovariance and Lorentz covariance of the differential calculus on $\kappa$-Minkowski space. These conditions imply that

(1) $\left[\hat{x}_{\mu}, \hat{\xi}_{\nu}\right]$ and $\left[\hat{x}_{\mu}, \theta^{\prime}\right]$ are closed in the vector space spanned by one-forms alone,

(2) all graded Jacobi identities in $\mathfrak{D}$ hold, 
(3) the action of the Lorentz algebra $\mathfrak{s o}(1, n-1)$ is covariant:

$$
\begin{aligned}
& M \triangleright\left(\hat{x}_{\mu} \hat{\xi}_{\nu}\right)=\left(M_{(1)} \triangleright \hat{x}_{\mu}\right) \hat{d}\left(M_{(2)} \triangleright \hat{x}_{\nu}\right), \\
& M \triangleright\left(\hat{\xi}_{\mu} \hat{x}_{\nu}\right)=\hat{d}\left(M_{(1)} \triangleright \hat{x}_{\mu}\right)\left(M_{(2)} \triangleright \hat{x}_{\nu}\right), \quad M \triangleright \theta^{\prime}=0,
\end{aligned}
$$

where $\hat{d}$ is the exterior derivative and $M$ is a generator of $\mathfrak{s o}(1, n-1)$. Here, the commutation relations in $\mathfrak{s o}(1, n-1)$ are undeformed,

$$
\left[M_{\mu \nu}, M_{\lambda \rho}\right]=\eta_{\nu \lambda} M_{\mu \rho}-\eta_{\mu \lambda} M_{\nu \rho}-\eta_{\nu \rho} M_{\mu \lambda}+\eta_{\mu \rho} M_{\nu \lambda}
$$

and $\Delta M=M_{(1)} \otimes M_{(2)}$ is the coproduct of $M$ in Sweedler notation:

$$
\begin{aligned}
& \Delta M_{i 0}=M_{i 0} \otimes 1+e^{a_{0} p_{0}} \otimes M_{i 0}-a_{0} \sum_{j=1}^{n-1} p_{j} \otimes M_{i j}, \\
& \Delta M_{i j}=M_{i j} \otimes 1+1 \otimes M_{i j},
\end{aligned}
$$

where $p_{\mu}$ is the momentum generator. The coproduct of the momentum generators is given by [7], 14]

$$
\begin{aligned}
& \Delta p_{0}=p_{0} \otimes 1+1 \otimes p_{0}, \\
& \Delta p_{i}=p_{i} \otimes 1+e^{a_{0} p_{0}} \otimes p_{i} .
\end{aligned}
$$

Relations (8)-(11) describe the coalgebra structure of the $\kappa$-Poincaré algebra generated by $M_{\mu \nu}$ and $p_{\mu}$. Note that Eq. (5) implies that the Lorentz generators act on a constant one-form by $M \triangleright \hat{\xi}_{\mu}=\hat{d}\left(M \triangleright \hat{x}_{\mu}\right)$.

In the following we shall briefly outline the construction of the algebra $\mathfrak{D}$ using realizations of $\hat{x}_{\mu}, \hat{\xi}_{\mu}$ and $\theta^{\prime}$ as formal power series in a Weyl super-algebra. Let $\mathcal{A}$ denote the unital associative algebra generated by commutative coordinates $x_{\mu}$, differential operators $\partial_{\mu}=\frac{\partial}{\partial x_{\mu}}$ and ordinary one-forms $d x_{\mu}$ satisfying the defining relations

$$
\begin{aligned}
{\left[x_{\mu}, x_{\nu}\right] } & =\left[\partial_{\mu}, \partial_{\nu}\right]=0, & {\left[\partial_{\mu}, x_{\nu}\right] } & =\eta_{\mu \nu} \\
{\left[d x_{\mu}, x_{\nu}\right] } & =\left[d x_{\mu}, \partial_{\nu}\right]=0, & \left\{d x_{\mu}, d x_{\nu}\right\} & =0 .
\end{aligned}
$$

Here, $\{$,$\} denotes the anticommutator and \eta=\operatorname{diag}(-1,1, \ldots, 1)$ is the Minkowski metric. $\mathcal{A}$ becomes a Weyl super-algebra if we define a graded commutator

$$
[[u, v]]=u v-(-1)^{|u||v|} v u
$$


where $|u|$ denotes the degree of a homogeneous element $u \in \mathcal{A}$. The degrees of the generators are defined by $\left|x_{\mu}\right|=\left|\partial_{\mu}\right|=0$ and $\left|d x_{\mu}\right|=1$. In this paper we consider two types of realizations of $\hat{x}_{\mu}$, the natural [44] and noncovariant [45, [46]. Following the notation in Ref. 14 the variables used in the natural and noncovariant realizations are denoted by $\left(X_{\mu}, D_{\mu}\right)$ and $\left(x_{\mu}, \partial_{\mu}\right)$, respectively. The reason for using different notation for the generators of $\mathcal{A}$ is that there exists an invertible transformation $\left(x_{\mu}, \partial_{\mu}\right) \mapsto\left(X_{\mu}, D_{\mu}\right)$ mapping the noncovariant into natural realization. The natural realization is defined by

$$
\hat{x}_{\mu}=X_{\mu} Z^{-1}-i a_{0} X_{0} D_{\mu}
$$

where $Z$ is invertible operator given by

$$
Z^{-1}=i a_{0} D_{0}+\sqrt{1+a_{0}^{2} D^{2}}
$$

The scalar product in (16) is taken with respect to the Minkowski metric, i.e. $D^{2}=$ $-D_{0}^{2}+\sum_{k=1}^{n-1} D_{k}^{2}$. $Z$ is called the shift operator because conjugation of $\hat{x}_{\mu}$ by $Z$ yields $Z \hat{x}_{\mu} Z^{-1}=\hat{x}_{\mu}+i a_{0} \delta_{0 \mu}$. One easily checks that the space-time coordinates represented by (15) satisfy the commutation relations (11). The realization (15) is a special case of covariant realizations of $\kappa$-Minkowski space introduced in Ref. [44].

Exterior derivative $\hat{d}$ is defined by $\hat{d}=\sum_{\alpha, \beta=0}^{n-1} k_{\alpha \beta}(D) d X_{\alpha} D_{\beta}$ where $k_{\alpha \beta}(D)$ is a formal power series in $a_{0}$ with coefficients in the ring of differential operators $D_{\mu}$. We require that $\lim _{a_{0} \rightarrow 0} \hat{d}=d$ where $d=-d X_{0} D_{0}+\sum_{k=1}^{n-1} d X_{k} D_{k}$ is the classical exterior derivative. The exterior derivative acts on space-time coordinates by $\hat{d} \cdot \hat{x}_{\mu}=\left[\left[\hat{d}, \hat{x}_{\mu}\right]\right]$. We define a noncommutative version of one-forms by $\hat{\xi}_{\mu}=\hat{d} \cdot \hat{x}_{\mu}$. Using relations (12)-(13) we find

$$
\lim _{a_{0} \rightarrow 0} \hat{\xi}_{\mu}=\left[d, X_{\mu}\right]=d X_{\mu}
$$

hence $\hat{\xi}_{\mu}$ is a deformation of ordinary one-form $d X_{\mu}$. Before proceeding further let us point out some general properties of a differential algebra constructed in this way:

(1) $\hat{d}$ satisfies the undeformed Leibniz rule

$$
\hat{d} \cdot(f(\hat{x}) g(\hat{x}))=(\hat{d} \cdot f(\hat{x})) g(\hat{x})+f(\hat{x})(\hat{d} \cdot g(\hat{x}))
$$

where $f(\hat{x})$ and $g(\hat{x})$ are monomials in $\hat{x}_{\mu}$.

(2) one-forms are closed, i.e. $\hat{d} \cdot \hat{\xi}_{\mu}=\left[\left[\hat{d}, \hat{\xi}_{\mu}\right]\right]=0$,

(3) one-forms anticommute, $\left\{\hat{\xi}_{\mu}, \hat{\xi}_{\nu}\right\}=0$, 
(4) the commutator for $\hat{\xi}_{\mu}$ and $\hat{x}_{\nu}$ is given by

$$
\left[\hat{\xi}_{\mu}, \hat{x}_{\nu}\right]=\sum_{\alpha=0}^{n-1} K_{\mu \nu}^{\alpha}(D) \hat{\xi}_{\alpha}
$$

where $K_{\mu \nu}^{\alpha}(D)$ generally depends on the differential operators $D_{\mu}$. If $K_{\mu \nu}^{\alpha}$ are constant for all values of $\mu, \nu$ and $\alpha$, then the differential algebra is closed.

We note that the Jacobi identity for $\hat{d} \cdot\left[\hat{x}_{\mu}, \hat{x}_{\nu}\right]=\left[\hat{d},\left[\hat{x}_{\mu}, \hat{x}_{\nu}\right]\right]$ together with commutation relations (10) implies that $\hat{x}_{\mu}$ and $\hat{\xi}_{\mu}$ satisfy the compatibility condition

$$
\left[\hat{\xi}_{\mu}, \hat{x}_{\nu}\right]-\left[\hat{\xi}_{\nu}, \hat{x}_{\mu}\right]=i\left(a_{\mu} \hat{x}_{\nu}-a_{\nu} \hat{\xi}_{\mu}\right)
$$

where $a_{\mu}=a_{0} \delta_{0 \mu}$. Extension of the above construction to higher order forms was presented in detail in Ref. [13].

Given the realization (15) we want to find a realization of $\hat{d}$ such that the action of $\hat{d}$ on $\hat{x}_{\mu}$ generates one-forms $\hat{\xi}_{\mu}$ and $\theta^{\prime}$ which close the algebra (2)-(4). Consider the following ansatz for $\hat{d}$ :

$$
\hat{d}=-d X_{0} D_{0}+\left(\sum_{k=1}^{n-1} d X_{k} D_{k}\right) Z .
$$

Substituting Eqs. (15) and (21) into $\hat{\xi}_{\mu}=\left[\left[\hat{d}, \hat{x}_{\mu}\right]\right]$ one finds

$$
\begin{aligned}
& \hat{\xi}_{0}=d X_{0}\left(Z^{-1}-i a_{0} D_{0}\right)+i a_{0}\left(\sum_{k=1}^{n-1} d X_{k} D_{k}\right) Z, \\
& \hat{\xi}_{k}=d X_{k}-i a_{0} d X_{0} D_{k} .
\end{aligned}
$$

The commutation relations for $\hat{x}_{\mu}$ and $\hat{\xi}_{\mu}$ are given by

$$
\begin{array}{llrl}
{\left[\hat{\xi}_{0}, \hat{x}_{0}\right]} & =-i a_{0} d X_{0} Z^{-1}+i a_{0} \hat{\xi}_{0}, & {\left[\hat{\xi}_{0}, \hat{x}_{j}\right]} & =i a_{0} \hat{\xi}_{j}, \\
{\left[\hat{\xi}_{i}, \hat{x}_{0}\right]} & =0, & {\left[\hat{\xi}_{i}, \hat{x}_{j}\right]=-i a_{0} \delta_{i j} d X_{0} Z^{-1} .}
\end{array}
$$

Note that the algebra (24)-(25) is not closed since the commutators involve an additional term $d X_{0} Z^{-1}$ which does not correspond to any one-form $\hat{\xi}_{\mu}$. However, the algebra can be closed by defining an extra one-form by $\theta^{\prime}=d X_{0} Z^{-1}$. Then one easily finds

$$
\left[\theta^{\prime}, \hat{x}_{0}\right]=-i a_{0} \theta^{\prime}, \quad\left[\theta^{\prime}, \hat{x}_{j}\right]=0 .
$$

The commutation relations (24)-(26) agree with the differential algebra (2)-(4). Thus, in our approach the extra cotangent direction $\theta^{\prime}$ introduced in Refs. 7] and [15] appears as a deformation of one-form $d X_{0}$ associated with time coordinate. In fact, $\hat{\xi}_{0}$ and $\theta^{\prime}$ are both deformations of $d X_{0}$, albeit different. 
2.1. Differential algebras of classical dimension. Different realizations of $\hat{x}_{\mu}$ and $\hat{d}$ lead to different differential calculi on $\kappa$-Minkowski space $\mathcal{M}_{\kappa}$. In the following we construct two families of differential algebras on $\mathcal{M}_{\kappa}$ such that there is a one-to-one correspondence between deformed one-forms and space-time coordinates. In both cases the realization of $\hat{x}_{\mu}$ is the same, but we consider two different deformations of the exterior derivative $\hat{d}$.

Let

$$
\hat{x}_{0}=x_{0}+i a_{0} \sum_{k=1}^{n-1} x_{k} \partial_{k}, \quad \hat{x}_{k}=x_{k} .
$$

This is a special case of noncovariant realizations of the algebra (1) introduced in Ref. [45], [46]. The transformation of variables $\left(x_{\mu}, \partial_{\mu}\right) \mapsto\left(X_{\mu}, D_{\mu}\right)$ which maps the noncovariant into natural realization is given in Ref. [14]. The realization (27) corresponds to the bicrossproduct basis in Ref. [3]. The shift operator corresponding to realization (27) is given by

$$
Z=\exp (A), \quad A=-i a_{0} \partial_{0}
$$

Let us define the exterior derivative

$$
\hat{d}_{1}=d x_{0} \frac{Z^{c}-1}{i a_{0} c}+\left(\sum_{k=1}^{n-1} d x_{k} \partial_{k}\right) Z^{-1}, \quad c \neq 0,
$$

where for $c \rightarrow 0$ we have $\lim _{c \rightarrow 0} \hat{d}_{1}=-d x_{0} \partial_{0}+\left(\sum_{k=1}^{n-1} d x_{k} \partial_{k}\right) Z^{-1}$. The one-forms $\hat{\xi}_{\mu}=$ $\left[\left[\hat{d}_{1}, \hat{x}_{\mu}\right]\right]$ with space-time coordinates represented by (27) are given by

$$
\hat{\xi}_{0}=d x_{0} Z^{c}, \quad \hat{\xi}_{k}=d x_{k} Z^{-1}
$$

Using realizations (27) and (30) we find

$$
\left[\hat{\xi}_{0}, \hat{x}_{0}\right]=i a_{0} c \hat{\xi}_{0}, \quad\left[\hat{\xi}_{k}, \hat{x}_{0}\right]=-i a_{0} \hat{\xi}_{k}, \quad\left[\hat{\xi}_{\mu}, \hat{x}_{j}\right]=0
$$

We denote the differential algebra (31) by $\mathfrak{D}_{1}$. Similarly, if the exterior derivative is defined by

$$
\hat{d}_{2}=d x_{0} \frac{Z^{c}-1}{i a_{0} c}+\left(\sum_{k=1}^{n-1} d x_{k} \partial_{k}\right) Z^{c-1},
$$

then the one-forms $\hat{\xi}_{\mu}=\left[\left[\hat{d}_{2}, \hat{x}_{\mu}\right]\right]$ are found to be

$$
\hat{\xi}_{0}=d x_{0} Z^{c}+i a_{0} c\left(\sum_{k=1}^{n-1} d x_{k} \partial_{k}\right) Z^{c-1}, \quad \hat{\xi}_{k}=d x_{k} Z^{c-1} .
$$


Now Eqs. (27) and (33) imply

$$
\left[\hat{\xi}_{0}, \hat{x}_{\mu}\right]=i a_{0} c \hat{\xi}_{\mu}, \quad\left[\hat{\xi}_{k}, \hat{x}_{0}\right]=i a_{0}(c-1) \hat{\xi}_{k}, \quad\left[\hat{\xi}_{k}, \hat{x}_{j}\right]=0
$$

The differential algebra (34) is denoted by $\mathfrak{D}_{2}$. Note that $\mathfrak{D}_{1}$ and $\mathfrak{D}_{2}$ are two families of differential algebras depending on a real parameter $c$ obtained from a fixed realization of $\hat{x}_{\mu}$. They are compatible with $\kappa$-Minkowski space since they satisfy the compatibility condition (20) and all graded Jacobi identities for the generators of $\mathfrak{D}_{1}$ and $\mathfrak{D}_{2}$ hold. The commutator $\left[\hat{\xi}_{\mu}, \hat{x}_{\nu}\right]$ in both algebras is closed in the vector space spanned by $\hat{\xi}_{0}, \hat{\xi}_{1}, \ldots, \hat{\xi}_{n-1}$, hence $\mathfrak{D}_{1}$ and $\mathfrak{D}_{2}$ are differential algebras of classical dimension.

\section{Action of the Lorentz Algebra on $\mathfrak{D}_{1}$ AND $\mathfrak{D}_{2}$}

The aim of this section is to construct an action of the Lorentz algebra $\mathfrak{s o}(1, n-1)$ on the algebras $\mathfrak{D}_{1}$ and $\mathfrak{D}_{2}$. First, we define an action of $\mathfrak{s o}(1, n-1)$ on the subalgebra $\mathcal{M}_{\kappa}$ and then extend it to $\mathfrak{D}_{1}$ and $\mathfrak{D}_{2}$.

It is natural to consider extension of the $\kappa$-Minkowski space (11) by momentum operators $p_{\mu}$. If we take the realization (27) and define $p_{\mu}=-i \partial_{\mu}$, then $\hat{x}_{\mu}$ and $\partial_{\mu}$ generate a deformed Heisenberg algebra given by the relations (11) and

$$
\left[p_{\mu}, p_{\nu}\right]=0, \quad\left[p_{0}, \hat{x}_{\mu}\right]=i \delta_{o \mu}, \quad\left[p_{k}, \hat{x}_{0}\right]=i a_{0} p_{k}, \quad\left[p_{k}, \hat{x}_{j}\right]=-i \delta_{k j}
$$

Note that the deformation of the algebra (35) depends on the realization of the Minkowski coordinates $\hat{x}_{\mu}$. A large class of such deformations was found in Refs. [44, 45] and [46]. Similarly, the $\kappa$-Minkowski space can be extended by the Lorentz algebra such that the direct sum of vector spaces $\mathfrak{g}_{\kappa}=\mathcal{M}_{\kappa} \oplus \mathfrak{s o}(1, n-1)$ is a Lie algebra. It can be shown that the cross commutator $\left[M_{\mu \nu}, \hat{x}_{\lambda}\right]$, which must be linear in $M_{\mu \nu}$ and $\hat{x}_{\mu}$, is uniquely given by [45, 46]

$$
\begin{array}{ll}
{\left[M_{i 0}, \hat{x}_{0}\right]=-\hat{x}_{i}+i a_{0} M_{i 0},} & {\left[M_{i j}, \hat{x}_{0}\right]=0,} \\
{\left[M_{i 0}, \hat{x}_{k}\right]=-\delta_{i k} \hat{x}_{0}+i a_{0} M_{i k},} & {\left[M_{i j}, \hat{x}_{k}\right]=\delta_{j k} \hat{x}_{i}-\delta_{i k} \hat{x}_{j} .}
\end{array}
$$

The algebra (36)-(37) is a subalgebra of the DSR algebra obtained as a cross product extension of $\kappa$-Minkowski and $\kappa$-Poincaré algebras [47, [48]. Since the commutation relations (36)-(37) are unique, the extension of $\mathcal{M}_{\kappa}$ by $\mathfrak{s o}(1, n-1)$ is independent of the 
realization of $\hat{x}_{\mu}$. If the coordinates $\hat{x}_{\mu}$ are given by the noncovariant realization (27), then the Lorentz generators are represented by

$$
\begin{aligned}
& M_{i 0}=x_{i}\left(\frac{1-Z}{i a_{0}}+\frac{i a_{0}}{2} \Delta-\frac{2}{i a_{0}} \sinh ^{2}\left(\frac{1}{2} A\right) Z\right)-\left(x_{0}+i a_{0} \sum_{k=1}^{n-1} x_{k} \partial_{k}\right) \partial_{i}, \\
& M_{i j}=x_{i} \partial_{j}-x_{j} \partial_{i},
\end{aligned}
$$

where $Z$ is given by Eq. (28) and $\Delta=\sum_{k=1}^{n-1} \partial_{k}^{2}$ is the Laplace operator. The realization (38)-(39) is a special case of the noncovariant realizations of the Lorentz algebra found in Refs. [45], [46]. In the classical limit we have $\lim _{a_{0} \rightarrow 0} M_{\mu \nu}=x_{\mu} \partial_{\nu}-x_{\nu} \partial_{\mu}$, as required.

Given the commutation relations (11) and (36)-(37) we want to define an action $\mathbf{s o}(1, n-$ 1) $\times \mathcal{M}_{\kappa} \rightarrow \mathcal{M}_{\kappa}$. Let $U\left(\mathfrak{g}_{\kappa}\right)$ be the enveloping algebra of $\mathfrak{g}_{\kappa}$ and let the generators of $U\left(\mathfrak{g}_{\kappa}\right)$ act on $1 \in U\left(\mathfrak{g}_{\kappa}\right)$ by $\hat{x}_{\mu} \triangleright 1=\hat{x}_{\mu}$ and $M_{\mu \nu} \triangleright 1=0$. Now define

$$
M_{\mu \nu} \triangleright f(\hat{x})=\left[M_{\mu \nu}, f(\hat{x})\right] \triangleright 1
$$

where $f(\hat{x})$ is a monomial in $\mathcal{M}_{\kappa}$. Using relations (36)-(37) the commutator in (40) can be written as a linear combination of terms with $M_{\mu \nu}$, if any, pushed to the far right. Thus, the action (40) is the projection of $\left[M_{\mu \nu}, f(\hat{x})\right]$ to the subalgebra $\mathcal{M}_{\kappa}$. For example, the action on Minkowski coordinates yields

$$
M_{\mu \nu} \triangleright \hat{x}_{\lambda}=\eta_{\nu \lambda} \hat{x}_{\mu}-\eta_{\mu \lambda} \hat{x}_{\nu}
$$

For monomials of order two we find

$$
\begin{aligned}
& M_{i 0} \triangleright\left(\hat{x}_{0} \hat{x}_{k}\right)=-\hat{x}_{i} \hat{x}_{k}-i a_{0} \delta_{i k} \hat{x}_{0}-\delta_{i k} \hat{x}_{0}^{2}, \\
& M_{i 0} \triangleright\left(\hat{x}_{k} \hat{x}_{0}\right)=-\hat{x}_{k} \hat{x}_{i}-\delta_{i k} \hat{x}_{0}^{2}, \\
& M_{i 0} \triangleright\left(\hat{x}_{k} \hat{x}_{l}\right)=\delta_{i k} \hat{x}_{0} \hat{x}_{l}-\delta_{i l} \hat{x}_{k} \hat{x}_{0}+i a_{0}\left(\delta_{k l} \hat{x}_{i}-\delta_{i l} \hat{x}_{k}\right), \\
& M_{i j} \triangleright\left(\hat{x}_{0} \hat{x}_{k}\right)=\delta_{j k} \hat{x}_{0} \hat{x}_{i}-\delta_{i k} \hat{x}_{0} \hat{x}_{j}, \\
& M_{i j} \triangleright\left(\hat{x}_{k} \hat{x}_{0}\right)=\delta_{j k} \hat{x}_{i} \hat{x}_{0}-\delta_{i k} \hat{x}_{j} \hat{x}_{0}, \\
& M_{i j} \triangleright\left(\hat{x}_{k} \hat{x}_{l}\right)=\delta_{j k} \hat{x}_{i} \hat{x}_{l}-\delta_{i k} \hat{x}_{j} \hat{x}_{l}+\delta_{j k} \hat{x}_{k} \hat{x}_{i}-\delta_{i l} \hat{x}_{k} \hat{x}_{j} .
\end{aligned}
$$

The above result is the same as that obtained by Majid and Ruegg [3] using the covariance condition $M_{\mu \nu} \triangleright(a b)=\left(M_{\mu \nu_{(1)}} \triangleright a\right)\left(M_{\mu \nu_{(2)}} \triangleright b\right), a, b \in \mathcal{M}_{\kappa}$, where the coproduct $\Delta M_{\mu \nu}=M_{\mu \nu_{(1)}} \otimes M_{\mu \nu_{(2)}}$ is given by Eqs. (8) -(99) and the momentum operator in (마) acts by $p_{\mu} \triangleright \hat{x}_{\nu}=-i \eta_{\mu \nu}$. This makes $\mathcal{M}_{\kappa}$ into an $\mathfrak{s o}(1, n-1)$-module algebra. 
Next, we want to extend the action of $\mathfrak{s o}(1, n-1)$ to the differential algebras $\mathfrak{D}_{1}$ and $\mathfrak{D}_{2}$ using the same prescription (401). For this purpose we need the commutator $\left[M_{\mu \nu}, \hat{\xi}_{\lambda}\right]$ where $\hat{\xi}_{\lambda} \in \mathfrak{D}_{1}$ or $\hat{\xi}_{\lambda} \in \mathfrak{D}_{2}$. One can show that the general form of this commutator is given by

$$
\left[M_{\mu \nu}, \hat{\xi}_{\lambda}\right]=\left[\hat{x}_{\mu}, \hat{\xi}_{\lambda}\right] \Phi_{\nu}-\left[\hat{x}_{\nu}, \hat{\xi}_{\lambda}\right] \Phi_{\mu}
$$

where $\Phi_{\mu}$ is a power series in $\partial_{\mu}$ such that $\Phi(0)=0$. The functions $\Phi_{\mu}$ depend on the realization of $M_{\mu \nu}$. If $M_{\mu \nu}$ is given by Eqs. (38)-(39), then

$$
\begin{aligned}
& \Phi_{0}=\frac{1-Z}{i a_{0}}+\frac{i a_{0}}{2} \Delta-\frac{2}{i a_{0}} \sinh ^{2}\left(\frac{1}{2} A\right) Z, \\
& \Phi_{k}=\partial_{k},
\end{aligned}
$$

where $\Delta=\sum_{k=1}^{n-1} \partial_{k}^{2}$. Since $\left[\hat{x}_{\mu}, \hat{\xi}_{\nu}\right] \in \operatorname{span}\left\{\hat{\xi}_{\mu} \mid 0 \leq \mu \leq n-1\right\}$, the commutator $\left[M_{\mu \nu}, \hat{\xi}_{\lambda}\right]$ depends only on momentum operators $p_{\mu}=-i \partial_{\mu}$ and one-forms $\hat{\xi}_{\lambda}$. This means that in order to extend the action of $\mathfrak{s o}(1, n-1)$ to $\mathfrak{D}_{k}$ we need to extend the algebra $U\left(\mathfrak{g}_{\kappa}\right)$ by the generators $p_{\mu}$ and $\hat{\xi}_{\mu}$ where the extension depends on whether $\hat{\xi}_{\mu} \in \mathfrak{D}_{1}$ or $\hat{\xi}_{\mu} \in \mathfrak{D}_{2}$. Denote the extended algebras by $\mathfrak{H}_{1}$ and $\mathfrak{H}_{2}$, respectively. Then $\mathfrak{H}_{k}$ contains $\mathfrak{D}_{k}$ and $\mathfrak{s o}(1, n-1)$ as Lie subalgebras, as well as the abelian algebra of translations generated by $p_{\mu}$. Define the action of $p_{\mu}$ and $\hat{\xi}_{\mu}$ on $1 \in \mathfrak{H}_{k}$ by $p_{\mu} \triangleright 1=0$ and $\hat{\xi}_{\mu}>1=\hat{\xi}_{\mu}$. Now we may define the action $\triangleright: \mathfrak{s o}(1, n-1) \times \mathfrak{D}_{k} \rightarrow \mathfrak{D}_{k}$ by

$$
M_{\mu \nu} \triangleright f(\hat{x}, \hat{\xi})=\left[M_{\mu \nu}, f(\hat{x}, \hat{\xi})\right] \triangleright 1
$$

where $f(\hat{x}, \hat{\xi})$ is a monomial in $\mathfrak{D}_{k}, k=1,2$. The action (51) is uniquely fixed by the commutation relations in $\mathfrak{D}_{k}$ and Eqs. (36)-(37) and (48). Since the generators of $\mathfrak{H}_{k}$ are constructed as elements of an associative algebra all Jacobi identities in $\mathfrak{H}_{k}$ hold. The Jacobi relations for $M_{\mu \nu}, \hat{x}_{\mu}$ and $\hat{\xi}_{\mu}$ guarantee that the action (51) is compatible with the commutation relations in $\mathfrak{D}_{k}$. Any monomial in $\mathfrak{D}_{k}$ can be written as a finite sum $f(\hat{x}, \hat{\xi})=\sum f_{1}(\hat{x}) f_{2}(\hat{\xi})$, hence it suffices to consider the action (51) on the products $f_{1}(\hat{x}) f_{2}(\hat{\xi})$. Since $\Phi_{\mu}>1=0$, Eq. (48) implies that the action of $M_{\mu \nu}$ on one-forms is trivial, i.e. $M_{\mu \nu} \triangleright f(\hat{\xi})=0$ for any monomial $f(\hat{\xi})$. Consequently, the action of $M_{\mu \nu}$ on product of monomials $f_{1}(\hat{x}) f_{2}(\hat{\xi})$ is given by

$$
M_{\mu \nu} \triangleright f_{1}(\hat{x}) f_{2}(\hat{\xi})=\left(M_{\mu \nu} \triangleright f_{1}(\hat{x})\right) f_{2}(\hat{\xi}) .
$$

The construction outlined here has the advantage that the action (51) is compatible with the algebra structure of $\mathfrak{D}_{1}$ and $\mathfrak{D}_{2}$ without introducing the extra one-form $\theta$ as in Ref. 
DIFFERENTIAL ALGEBRAS ON $\kappa$-MINKOWSKI SPACE AND ACTION OF THE LORENTZ ALGEBRA1

[7. However, the action is not Lorentz covariant since the necessary condition $M_{\mu \nu}$

$\hat{\xi}_{\lambda}=\hat{d} \cdot\left(M_{\mu \nu} \hat{x}_{\lambda}\right)$ does not hold. Indeed, in view of Eq. (41) we have $\hat{d} \cdot\left(M_{\mu \nu} \triangleright \hat{x}_{\lambda}\right)=$ $\eta_{\nu \lambda} \hat{\xi}_{\mu}-\eta_{\mu \lambda} \hat{\xi}_{\nu}$ while $M_{\mu \nu} \triangleright \hat{\xi}_{\lambda}=0$. This problem can be partially resolved by modifying the realization of $M_{\mu \nu}$ such that $M_{\mu \nu}-\hat{\xi}_{\lambda}=\eta_{\nu \lambda} \hat{\xi}_{\mu}-\eta_{\mu \lambda} \hat{\xi}_{\nu}$ is satisfied. This modification of (51) if given as follows.

Consider extension $\tilde{\mathcal{A}}$ of the algebra (12)-(13) by a set of generators $q_{\mu}$ subject to defining relations

$$
\left[x_{\mu}, q_{\nu}\right]=\left[\partial_{\mu}, q_{\nu}\right]=0, \quad\left\{q_{\mu}, q_{\nu}\right\}=0, \quad\left\{d x_{\mu}, q_{\nu}\right\}=\eta_{\mu \nu}
$$

The degree of $q_{\mu}$ is defined to be $\left|q_{\mu}\right|=1$. Note that the variables $q_{\mu}$ play the role of a Grassmann type derivative with respect to one-forms $d x_{\mu}$. The $\kappa$-deformed superHeisenberg algebra, generated by $\hat{x}_{\mu}, \partial_{\mu}, \hat{\xi}_{\mu}$ and $q_{\mu}$, satisfies all graded Jacobi identities. Let us define

$$
M_{\mu \nu}^{(1)}=d x_{\mu} q_{\nu}-d x_{\nu} q_{\mu},
$$

and let

$$
\widetilde{M}_{\mu \nu}=M_{\mu \nu}+M_{\mu \nu}^{(1)}
$$

where the Lorentz generators $M_{\mu \nu}$ are given by the realization (38)-(39). It is easily seen that $M_{\mu \nu}^{(1)}$ close the relations (7) and $\left[M_{\mu \nu}, M_{\lambda \rho}^{(1)}\right]=0$. Consequently, $\widetilde{M}_{\mu \nu}$ also satisfy the relations (77), hence Eq. (55) represents a new realization of $\mathfrak{s o}(1, n-1)$ in terms of the extended algebra $\tilde{\mathcal{A}}$. To this realization of $\mathfrak{s o}(1, n-1)$ we associate the action

$$
\widetilde{M}_{\mu \nu} \triangleright f(\hat{x}, \hat{\xi})=\left[\widetilde{M}_{\mu \nu}, f(\hat{x}, \hat{\xi})\right] \triangleright 1
$$

The action is consistent with the commutation relations in $\mathfrak{D}_{1}$ and $\mathfrak{D}_{2}$ since all Jacobi identities for $\widetilde{M}_{\mu \nu}, \hat{x}_{\mu}$ and $\hat{\xi}_{\mu}$ are satisfied. For products of monomials $f_{1}(\hat{x}) f_{2}(\hat{\xi})$ the action (56) satisfies the Leibniz-like rule

$$
\widetilde{M}_{\mu \nu} \triangleright f_{1}(\hat{x}) f_{2}(\hat{\xi})=\left(M_{\mu \nu} \triangleright f_{1}(\hat{x})\right) f_{2}(\hat{\xi})+f_{1}(\hat{x})\left(M_{\mu \nu}^{(1)} \triangleright f_{2}(\hat{\xi})\right)
$$

where $M_{\mu \nu}$ acts only on coordinates $\hat{x}_{\mu}$ and $M_{\mu \nu}^{(1)}$ acts only on one-forms $\hat{\xi}_{\mu}$. It follows from Eq. (157) that $\widetilde{M}_{\mu \nu} \triangleright f_{1}(\hat{x})=M_{\mu \nu} \triangleright f_{1}(\hat{x})$, hence the actions (51) and (56) agree on the $\kappa$-Minkowski space. In particular, we have the vector-like transformation $\widetilde{M}_{\mu \nu} \hat{x}_{\lambda}=\eta_{\nu \lambda} \hat{x}_{\mu}-\eta_{\mu \lambda} \hat{x}_{\nu}$. On the other hand, the action of $\widetilde{M}_{\mu \nu}$ on one-forms is 
nontrivial since one-forms also transform vector-like, $\widetilde{M}_{\mu \nu} \triangleright \hat{\xi}_{\lambda}=\eta_{\nu \lambda} \hat{\xi}_{\mu}-\eta_{\mu \lambda} \hat{\xi}_{\nu}$. This implies

$$
\widetilde{M}_{\mu \nu} \triangleright \hat{\xi}_{\lambda}=\hat{d} \cdot\left(\widetilde{M}_{\mu \nu} \triangleright \hat{x}_{\lambda}\right)
$$

thus the action is Lorentz covariant on constant one-forms. Restriction of (56) to monomials in $\hat{\xi}_{\mu}$ satisfies the ordinary Leibniz rule

$$
\widetilde{M}_{\mu \nu} \triangleright f(\hat{\xi}) g(\hat{\xi})=\left(\widetilde{M}_{\mu \nu} \triangleright f(\hat{\xi})\right) g(\hat{\xi})+f(\hat{\xi})\left(\widetilde{M}_{\mu \nu} \triangleright g(\hat{\xi})\right) .
$$

Using Eq. (57) and the rules for computing $M_{\mu \nu} \triangleright f_{1}(\hat{x})$ and $M_{\mu \nu}^{(1)} \triangleright f_{2}(\hat{\xi})$ one can easily calculate the action of $\widetilde{M}_{\mu \nu}$ on arbitrary monomials $f(\hat{x}, \hat{\xi}) \in \mathfrak{D}_{k}, k=1,2$. For example,

$$
\widetilde{M}_{\mu \nu} \triangleright \hat{x}_{\lambda} \hat{\xi}_{\rho}=\left(\eta_{\nu \lambda} \hat{x}_{\mu}-\eta_{\mu \lambda} \hat{x}_{\nu}\right) \hat{\xi}_{\rho}+\hat{x}_{\lambda}\left(\eta_{\nu \rho} \hat{\xi}_{\mu}-\eta_{\mu \rho} \hat{\xi}_{\nu}\right) .
$$

The condition (58) does not extend by Lorentz covariance to entire algebras $\mathfrak{D}_{1}$ and $\mathfrak{D}_{2}$. This is in accordance with the theory developed in Ref. [7] since otherwise this would be in contradiction with the Jacobi identity for $\hat{x}_{\mu}, \hat{x}_{\nu}$ and $\hat{\xi}_{\lambda}$ (for more details see Ref. [7]).

\section{Conclusion}

In this paper we have constructed differential algebras on $\kappa$-Minkowski space-time using realizations of coordinates $\hat{x}_{\mu}$ and one-forms $\hat{\xi}_{\mu}$ as formal power series in a Weyl superalgebra. The algebras considered here are the well-known differential algebra introduced by Sitarz [7] as well as new families of differential algebras $\mathfrak{D}_{1}$ and $\mathfrak{D}_{2}$. The algebras $\mathfrak{D}_{1}$ and $\mathfrak{D}_{2}$ are obtained from a fixed realization of $\hat{x}_{\mu}$ and using different realization of exterior derivative $\hat{d}$. The resulting one-forms $\hat{\xi}_{\mu}=\left[\left[\hat{d}, \hat{x}_{\mu}\right]\right]$ have the property that the commutator $\left[\hat{\xi}_{\mu}, \hat{x}_{\nu}\right]$ is closed in the vector space spanned by $\hat{\xi}_{0}, \hat{\xi}_{1}, \ldots, \hat{\xi}_{n-1}$ alone. We have also presented a novel construction of an action of $\mathfrak{s o}(1, n-1)$ on $\mathfrak{D}_{1}$ and $\mathfrak{D}_{2}$ using realizations of the Lorentz generators in terms of Grassmann-type variables $q_{\mu}$. The action does not require introduction of an extra cotangent direction $\theta$ as in Ref. [7]. When restricted to Minkowski coordinates, $\mathfrak{s o}(1, n-1)$ acts covariantly on the $\kappa$-Minkowski space making it into an $\mathfrak{s o}(1, n-1)$-module algebra. The Lorentz covariance is valid for constant one-forms but it does not extend to entire algebras $\mathfrak{D}_{1}$ and $\mathfrak{D}_{2}$. In this approach there is a one-to-one correspondence between the Minkowski coordinates and one-forms. This provides a certain advantage since every variable in the noncommutative setting is for a given realization a unique deformation of the corresponding classical variable. In this 
paper we have focused only on the action of the Lorentz algebra generated by $M_{\mu \nu}$ and $\widetilde{M}_{\mu \nu}$ (Eqs. (38), (39), (54) and (55)), but there are also other implementations of Lorentz algebras compatible with the $\kappa$-Minkowski space-time (Refs. [11, [12], [49] and [50]). Further developments of this approach as well as its applications to field theory, statistics, twist operators (see Refs. [12], [42], [43] and [49]) and dispersion relations [51] will be presented elsewhere.

\section{ACKNOWLEDGEMENTS}

This work was supported by the Ministry of Science and Technology of the Republic of Croatia under contract No. 098-0000000-2865 and 177-0372794-2816.

\section{REFERENCES}

[1] A. Connes, Noncommutative Geometry, Academic Press, 1994.

[2] S. Majid, Foundations of Quantum Group Theory, Cambridge University Press, 1995.

[3] S. Majid and H. Ruegg, "Bicrossproduct structure of $\kappa$-Poincaré group and noncommutative geometry", Phys. Lett. B 334, 348 (1994), hep-th/9404107

[4] S. L. Woronowicz, "Differential Calculus on Compact Matrix Pseudogroups (Quantum Groups)", Comm. Math. Phys. 122, 125 (1989).

[5] P. Aschieri, F. Lizzi and P. Vitale, "Twisting all the way: from classical to quantum mechanics", Phys. Rev. D 77 025037, (2008), arXiv:0708.3002 2 .

[6] G. Landi, An Introduction to Noncommutative Spaces and Their Geometries, Lect. Notes Phys, m 51 (1997), hep-th/9701078v1.

[7] A. Sitarz, "Noncommutative differential calculus on the kappa-Minkowski space", Phys. Lett. B 349, 42 (1995), hep-th/9409014.

[8] C. Gonera, P. Kosinski and P. Maslanka, "Differential calculi on quantum Minkowski space", J. Math. Phys. 37, 5820 (1996), arXiv:q-alg/9602007.

[9] M. Dimitrijević, L. Möller and E. Tsouchnika, "Derivatives, forms and vector fields on the $\kappa$-deformed Euclidean space", J. Phys. A: Math. Theor. 37 (2004), hep-th/0404224.

[10] J. Wess, "Deformed coordinates spaces; Derivatives", Lecture given at the Balkan workshop BW2003, August 2003, Vrnjačka Banja, Serbia, hep-th/0408080

[11] H. C. Kim, Y. Lee, C. Rim and J. H. Yee, "Differential structure on the $\kappa$-Minkowski spacetime from twist", Phys. Lett. B 671, 398 (2009), arXiv:hep-th/0808.2866.

[12] J.G. Bu, J.H. Yee and H.C. Kim, "Differential structure on $\kappa$-Minkowski spacetime realized as module of twisted Weyl algebra", Phys. Lett. B 679, 486 (2009), arXiv:0903.0040v2.

[13] S. Meljanac and S. Krešić-Jurić, "Noncommutative differential forms on the kappa-deformed space", J. Phys. A: Math. Theor. 42, 365204 (2009), arXiv:0804.3072. 
[14] S. Meljanac and S. Krešić-Jurić, "Differential structure on $\kappa$-Minkowski space, and $\kappa$-Poincaré algebra", Int. J. Mod. Phys. A 26 (20), 3385 (2011), arXiv: 1004.4547.

[15] S. Majid, "Quantum Anomalies and Newtonian Gravity on Quantum Spacetime", arXiv:1109.6190v1.

[16] J. Lukierski, A. Nowicki, H. Ruegg and V. N. Tolstoy, "Q-deformation of Poincaré algebra", Phys. Lett. B 264, 331 (1991).

[17] J. Lukierski, A. Nowicki and H. Ruegg, "New quantum Poincaré algebra, and $\kappa$-deformed field theory", Phys. Lett. B 293, 344 (1992).

[18] J. Lukierski and H. Ruegg, "Quantum $\kappa$-Poincaré in any dimension", Phys. Lett. B 329, 189 (1994), hep-th/9310117.

[19] J. Lukierski, M. Woronowicz, "New Lie algebraic and quadratic deformations of Minkowski space from twisted Poincaré symmetries", Phys. Lett. B 633, 116 (2006), hep-th/0508083

[20] P. Kosiński and P. Maślanka, "The duality between $\kappa$-Poincaré algebra and $\kappa$-Poincaré group", hep-th/9411033

[21] S. Zakrzewski, "Quantum Poincaré group related to the kappa-Poincaré algebra", J. Phys. A 27, 2075 (1994).

[22] G. Amelino-Camelia and M. Arzano, "Coproduct and star-product in field theories on Lie algebra noncomutative spacetime", Phys. Rev.D 65, 084044 (2002), hep-th/0105120

[23] L. Möller, "A symmetry invariant integral on $\kappa$-deformed spacetime", JHEP 0512, 029 (2005), hep-th/0409128

[24] A. Agostini, G. Amelino-Camelia, M. Arzano, A. Marciano and R.A. Tacchi, "Generalizing the Noether theorem for Hopf-algebra spacetime symmetries”, Mod. Phys. Lett. A22 1779-1786, (2007), hep-th/0607221.

[25] E. J. Beggs and S. Majid, "Semiclassical differential structures", Pac. J. Math. 224, 1 (2006).

[26] G. Amelino-Camelia, "Testable scenario for relativity with minimum-length", Phys. Lett. B 510, 255 (2001), hep-th/0012238.

[27] G. Amelino-Camelia, "Relativity in space-times with short-distance structure governed by an observerindependent (Planckian) length scale", Int. J. Mod. Phys. D 11, 35 (2002), gr-qc/0012051

[28] N. R. Bruno, G. Amelino-Camelia and J. Kowalski-Glikman, "Deformed boost transformations that saturate at the Planck scale", Phys. Lett. B 522, 133 (2001), hep-th/0107039

[29] J. Kowalski-Glikman and S. Nowak, "Double special relativity theories as different bases of kappaPoincaré algebra", Phys. Lett. B 539, 126 (2002), hep-th/0203040

[30] L. Freidel, J. Kowalski-Glikman and S. Nowak, "Field theory on $\kappa$-Minkowski space revisited: Noether charges and breaking of Lorentz symmetry", Int. J. Mod. Phys. A 23, 2687 (2008), arXiv:0706.3658

[31] J. Kowalski-Glikman, "Introduction to doubly special relativity", Lect. Notes Phys. 669, 131 (2005), hep-th/0405273v1.

[32] J. Kowalski-Glikman and S. Nowak, "Non-commutative space-time of doubly special relativity theories", Int. J. Mod. Phys. D 12, 299 (2003). 
[33] M. Daskiewicz, K. Imilkowska, J. Kowalski-Glikman and S. Nowak, "Scalar field theory on $\kappa$ Minkowski space-time and doubly special relativity", Int. J. Mod. Phys. A 20, 4925 (2005).

[34] M. Daszkiewicz, J. Lukierski and M. Woronowicz, "Towards Quantum Noncommutative $\kappa$-deformed Field Theory", Phys. Rev. D 77, 105007 (2008), arXiv:hep-th/0708.1561.

[35] P. Kosinski, J. Lukierski and P. Maslanka, "Local D=4 Field Theory on $\kappa$-Deformed Minkowski Space", Phys. Rev. D 62, 025004 (2000), arXiv:hep-th/9902037;

[36] H. C. Kim, Y. Lee, C. Rim and J. H. Yee, "Scalar field theory in $\kappa$-Minkowski spacetime from twist", J. Math. Phys. 50, 102304 (2009), arXiv:hep-th/0901.0049.

[37] S. Meljanac and A. Samsarov, "Scalar field theory on kappa-Minkowski spacetime and translation and Lorentz invariance", Int. J. Mod. Phys. A 26, 1439 (2011), arXiv:1007.3943.

[38] S. Meljanac, A. Samsarov, J. Trampetic and M. Wohlgenannt, "Scalar field propagation in the phi 4 kappa-Minkowski model", JHEP 1112, 010 (2011), arXiv:1111.5553.

[39] E. Harikumar, T. Juric and S. Meljanac, "Electrodynamics on $\kappa$-Minkowski space-time", Phys. Rev. D 84, 085020 (2011), arXiv:1107.3936.

[40] E. Harikumar, "Maxwell's equations on the $\kappa$-Minkowski spacetime and Electric-Magnetic duality", Europhys. Lett. 90, 21001 (2010), arXiv:1002.3202 v3.

[41] M. Dimitrijevic and L. Jonke, "A twisted look on kappa-Minkowski: U(1) gauge theory", JHEP 1112, 080 (2011), arXiv:1107.3475.

[42] T. R. Govindarajan, K. S. Gupta, E. Harikumar, S. Meljanac and D. Meljanac, "Twisted statistics in $\kappa$-Minkowski spacetime", Phys. Rev. D 77, 105010 (2008), arXiv:0802.1576.

[43] T. R. Govindarajan, K. S. Gupta, E. Harikumar, S. Meljanac and D. Meljanac, "Deformed osciallator algebras and QFT in the $\kappa$-Minkowski spacetime", Phys. Rev. D 80, 025014 (2009), arXiv:0903.2355.

[44] S. Meljanac, S. Krešić-Jurić and M. Stojić, "Covariant realizations of kappa-deformed space", Eur. Phys. J. C 51, 229 (2007).

[45] S. Meljanac and M. Stojić, "New realizations of Lie alegbra kappa-deformed Euclidean space", Eur. Phys. J. C 47, 531 (2006).

[46] S. Meljanac, A. Samsarov, M. Stojić and K. S. Gupta, "Kappa-Minkowski space-time and the star product realizations", Eur. Phys. J. C 53, 295 (2008), arXiv:0705.2471.

[47] A. Borowiec and A. Pachol, " $\kappa$-Minkowski Spacetimes and DSR Algebras: Fresh Look and Old Problems", SIGMA 6, 086 (2010), arXiv:1005.4429

[48] A. Borowiec and A. Pachol, "The classical basis for the $\kappa$-Poincaré Hopf algebra and doubly special relativity theories", J. Phys. A: Math. Theor. 43, 045203 (2010), arXiv:0903.5251

[49] A. Boroweic and A. Pachol, " $\kappa$-Minkowski spacetime as the result of Jordanian twist deformation", Phys. Rev. D 79, 045012 (2009), arXiv:0812.0576.

[50] D. Kovačević, S. Meljanac, A. Pachoł and R. Štrajn, "Generalized $\kappa$-Poincaré algebras, Hopf algebras and $\kappa$-Minkowski spacetime", arXiv:1202.3305v1.

[51] A. Borowiec, Kumar S. Gupta, S. Meljanac and A. Pachoł, "Constraints on the quantum gravity scale from kappa-Minkowski spacetime", Europhys. Lett. 92, 20006 (2010), arXiv:0912.3299. 
Rudjer Bošković Institute, BijeničKa Cesta B.B., 10000 Zagreb, Croatia

E-mail address: meljanac@irb.hr

Faculty of Natural and Mathematical Sciences, University of Split, Teslina 12, 21000 Split, Croatia

E-mail address: skresic@pmfst.hr

Rudjer Bošković Institute, Bijenička Cesta B.B., 10000 Zagreb, Croatia

E-mail address: rina.strajn@gmail.com 